\title{
Stratigraphische und geomorphologische Auswertung von Schuttdecken vor Muschelkalkschichtkämmen und -schichtstufen im niedersächsischen Bergland

\author{
Von Gerhard Bartels, Göttingen
}

\section{Mit 1 Abbildung}

$\mathrm{Z}$ us a mmenfassung. Die Schuttdecken vor einigen Strukturformen des Muschelkalkes im niedersächsischen Bergland werden stratigraphisch gedeutet. Sie bestehen aus Muschelkalkwanderschutt und Rötfließerden und enthalten in ihrem unteren Teil lagenweise eine Lößbeimengung. Innerhalb dieser älteren Schichten lassen sich häufig Schwemmsedimente feststellen. Die Schuttdecken können aufgrund ihrer Beziehung zu den Talböden der letzten Kaltzeit und aufgrund anderer Kriterien in das Weichselglazial gestellt werden. Thre Auswertung für die Geomorphologie ergibt, daß der Schwerpunkt der Abtragung während der letzten Kaltzeit in der Nähe der Röt-Muschelkalk-Grenze lag, so daß sich die Durchbiegung des Hanges vergrößerte. Das an Strukturformen des Muschelkalkes häufig auftretende konkave Vorderseitenprofil läßt sich also auf pleistozän-periglaziale Prozesse zurückführen.

$\mathrm{S} \mathrm{u} \mathrm{mm}$ a r y. The subject of this research are slope sediments in front of ridges and cuestas built up by Triassic limestone (Muschelkalk) in the hilly southern part of Lower Saxony. The stratigraphical and geomorphological significance of the different layers is explained. The young Würm loess covers a limestone débris, generally without admixture of loess, or a series of limestone débris/clayey Röth detritus. These layers caused by solifluction, overlay a waste (limestone and Röth detritus), which in some horizons shows a remarkable content of loess. By analysing the sedimentary type of these older layers slope-wash débris frequently could be detected.

The mentioned slope sediments accumulated during the Weichselian period. Their geomorphological interpretation shows that under periglacial conditions the slopes retreated rather intensively near the limiting surface between Röth and Muschelkalk. Thus the frontal slopes got a typical concave bend.

Bei geomorphologischen Feldarbeiten für eine Dissertation über den Hildesheimer Wald wurden die Schuttdecken vor einigen Schichtkämmen des Unteren Muschelkalkes genauer untersucht. Diese Schuttdecken sollen mit Hangsedimenten vor anderen Strukturformen des Muschelkalkes im niedersächsischen Bergland verglichen werden.

Die erste der drei im Hildesheimer Wald angelegten Grabungsreihen (Abb. 1) schließt die Lockersedimente eines konkaven Stirnhanges auf, der typisch für fast alle markanten

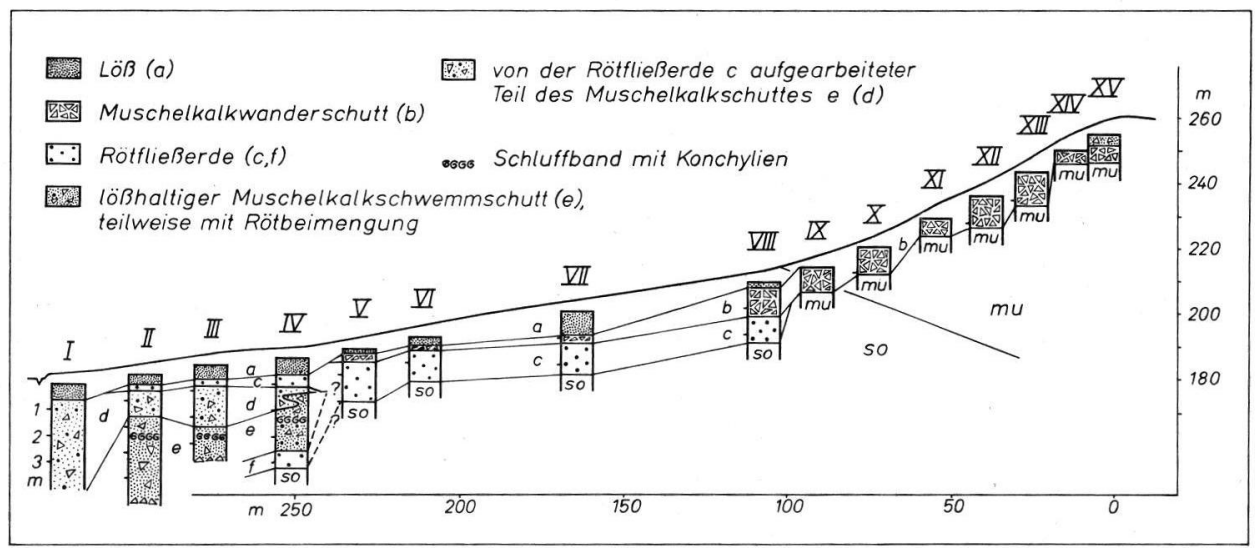

Abb. 1. Die Schuttdecken eines konkaven Wellenkalkstirnhanges

(Triesberg im Hildesheimer Wald). 
Muschelkalkkämme ist. Der unter dem Solifluktionslöß (a) folgende Muschelkalkschutt (b) und das Rötmaterial (c, f) lassen sich aufgrund ihres Fließgefüges als solifluidale Bildungen ansprechen. Der Kalkschutt (e) erscheint jedoch schon bei makroskopischer Beobachtung nicht als Solifluktionshorizont, denn er enthält an seiner Basis gebänderte Schlufflagen, die sich nur als aquatische Bildungen deuten lassen. Der Verdacht liegt daher nahe, daß der gesamte Schutt (e) ein Schwemmsediment darstellt. Diese Vermutung läßt sich dadurch nachprüfen, daß man die Einregelung der Längsachsen und die Kornverteilung des fraglichen Kalkschuttes (e) mit der des Wanderschuttes (b) vergleicht.

Die Mehrzahl der Längsachsen des Wanderschuttes weist in Richtung des Hanggefälles (Gruppe I). Der Schicht (e) fehlt jedoch diese solifluidale Einregelung (Tab. 1). Sie läßt sich daher nicht als Wanderschutt deuten.

Tabe 11 e 1

Einregelung der Längsachsen

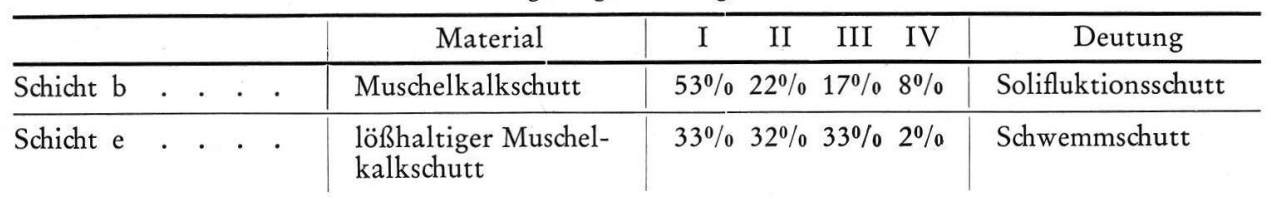

Auch die Kornverteilung der beiden Sedimente ist verschieden (Tab. 2). Der Wellenkalkwanderschutt besteht, wie häufig im niedersächsischen Bergland (ROHDENBurg \& MEYER 1963), vorwiegend aus Kieskomponenten und Feinmaterial (0-0,02 mm), während die Sandfraktionen fast ganz fehlen. Bei dem fraglichen Schutt (e) sind dagegen die mittleren Korngrößen selektiv angereichert. Diese Sortierung läßt sich nur auf aquatischen Transport zurückführen. Sie ist sogar noch größer, als es die Kornverteilung veranschaulicht, denn das karbonatische Feinmaterial wurde bei der Schicht (e) ausgewaschen und durch Löß ersetzt. Der ältere Kalkschutt ist folglich ein Schwemmschutt.

Tabe 11 e 2

Kornverteilung der Hangsedimente

(Angaben in $\%$, Summe $0-2 \mathrm{~mm}=100 \%$ )

\begin{tabular}{|c|c|c|c|c|}
\hline & & Schicht b & Schicht e & \\
\hline & $>2$ & 138,7 & 48,4 & \\
\hline 2 & $-0,5 \mathrm{~mm}$ & 6,4 & 9,2 & \\
\hline 0,5 & $-0,2 \mathrm{~mm}$ & 1,7 & 5,2 & \\
\hline & $-0,06 \mathrm{~mm}$ & 2,8 & 5,5 & \\
\hline 0,06 & $-0,02 \mathrm{~mm}$ & 14,5 & 42,0 & \\
\hline 0,02 & $-0,006 \mathrm{~mm}$ & 37,6 & 16,5 & \\
\hline 0,00 & $6-0,002 \mathrm{~mm}$ & 15,8 & 4,6 & \\
\hline 0,00 & $2-0 \quad \mathrm{~mm}$ & 21,2 & 17,0 & \\
\hline & arbonatgehalt & $57,8 \%$ & $13,4 \%$ & $(0-0,06 \mathrm{~mm})$ \\
\hline
\end{tabular}

Sowohl bei diesem Profil als auch an anderen Wellenkalkstirnhängen finden sich keine Spuren einer intensiven holozänen Abtragung. Die Lockersedimente lassen sich wegen dieses Mangels an rezenten Abtragungsspuren, wegen ihrer Solifluidaltextur und der Überlagerung durch Weichsellöß als pleistozän-kaltzeitlich ansprechen. Die Schuttdecken enthalten auch in dem Akkumulationsbereich des Unterhanges keine interglazialen Bodenbildungen. Außerdem tritt in dieser Grabungsreihe kein glazigenes Material auf, obwohl der Hildesheimer Wald innerhalb des saaleglazialen Vereisungsgebietes liegt. Schließlich ist für die Datierung der Schuttdecken wichtig, daß sie unter den weichselzeitlichen Talboden hinabziehen und sich teilweise mit dem Schwemmschutt des Talgrundes verzahnen. Die Hangsedimente sind aus diesen Gründen in die Weichselkaltzeit zu stellen. 
Tabelle 3

Stratigraphische Ubersicht über Schuttdeckenfolgen an Muschelkalkschichtkämmen im Hildesheimer Wald

\begin{tabular}{|c|c|c|}
\hline Grabungsreihe I & Grabungsreihe II & $\begin{array}{c}\text { Grabungsreihe III } \\
\text { Oberhang }\end{array}$ \\
\hline Solifluktionslöß & Solifluktionslöß & Solifluktionslöß \\
\hline $\begin{array}{l}\text { lößfreier Muschelkalkwander- } \\
\text { schutt }\end{array}$ & $\begin{array}{l}\text { lößfreier Muschelkalkwander- } \\
\text { schutt }\end{array}$ & \multirow{2}{*}{$\begin{array}{l}\text { lößfreier Muschelkalkwander- } \\
\text { schutt }\end{array}$} \\
\hline Rötfließerde & Rötfließerde & \\
\hline $\begin{array}{l}\text { lößhaltiger Muschelkalk- } \\
\text { schwemmschutt mit konchylien- } \\
\text { haltigem Schluffband, lagen- } \\
\text { weise Rötbröckchen }\end{array}$ & $\begin{array}{l}\text { Röt-Muschelkalk-Fließerde mit } \\
\text { eingeschalteter Lage lößhaltigen } \\
\text { Muschelkalkschwemmschuttes, } \\
\text { stellenweise moränisches } \\
\text { Material }\end{array}$ & \multirow[t]{2}{*}{$\begin{array}{l}\text { lößhaltiger Muschelkalk- } \\
\text { wanderschutt } \\
\text { (von Grundmoräne unterlagert) }\end{array}$} \\
\hline Rötfließerde & Rötfließerde & \\
\hline
\end{tabular}

Die Hangsedimente der 2. Grabungsreihe zeigen eine ähnliche Abfolge (Tab. 3). In dem 3. Profil (Abb. s. Bartels 1966) wurden nur auf dem oberen, flachen Teil des Oberhanges ältere Schuttdecken angeschürft, denen natürlich das Rötmaterial des Unterhanges fehlt. Diese Sedimente enthalten zwar moränisches Material, das aber mit Ausnahme der Grundmoräne der 3. Grabungsreihe solifluidal verlagert ist. Außerdem verzahnen sich die Schuttdecken mit weichselzeitlichen Talfüllungen, so daß sie in die letzte Kaltzeit zu stellen sind.

Leider fehlen in den Schuttdeckenfolgen interstadiale Böden oder andere Leitmarken. Die Vermutung, daß das Schneckenschalen führende Schluffband (Abb. 1) ein Interstadial verträte, konnte bisher nicht bestätigt werden. Die von Frau Dr. M. BRunNacker und Prof. Dr. K. BRUnNACKer freundlicherweise durchgeführten Molluskenuntersuchungen ergaben nur das Bruchstück einer Pupilla spec. Die anderen Bruchstücke ließen sich nicht bestimmen. Die einzelnen Lagen können aus diesen Gründen nur näherungsweise miteinander parallelisiert werden. Bei der Parallelisierung von Schuttdecken ist außerdem zu beachten, daß die Ablagerungsvorgänge nicht nur vom Klima, sondern auch von lokalen Faktoren (Länge und Neigung des Hanges, Exposition) beeinflußt werden. Es ist daher möglich, daß sich nicht jede Lage an allen Hängen gebildet oder erhalten hat. Die stratigraphischen Aspekte können daher nur zu einer Arbeitshypothese führen.

Die Lößdecke, der Muschelkalkwanderschutt und die Rötfließerde lassen sich von Profil zu Profil durch Bohrreihen miteinander verknüpfen und sind daher zweifelsohne gleichzeitige Bildungen. Wegen der gleichen Stellung in der Sedimentfolge liegt der Verdacht nahe, daß innerhalb der älteren Schuttdecken die basalen Rötfließerden einander entsprechen und daß die Röt-Muschelkalk-Fließerde des 2. Profils das Äquivalent des Schwemmschuttes in dem 1. darstellt (Tab. 3). Bezeichnenderweise ist der Röt-Muschelkalk-Fließerde eine Lage von lößhaltigem Muschelkalkschwemmschutt eingeschaltet. Eine weitere Gemeinsamkeit liegt darin, daß beide Sedimente Röt- und Muschelkalkmaterial enthalten. Sollen diese beiden Schichten einander entsprechen, so muß es eine Erklärung für ihre verschiedene Genese und Zusammensetzung geben. Die beiden Grabungsreihen unterscheiden sich vor allem durch ihren verschiedenen Oberhang. Man muß annehmen, daß der über $30^{\circ}$ steile und etwa $50 \mathrm{~m}$ hohe Oberhang des 1 . Profiles aufgrund seiner größeren Neigung und Ausdehnung erheblich mehr Muschelkalkmaterial geliefert hat als der wesentlich flachere Oberhang des 2. Profiles. Dadurch konnte der Unterhang der 1. Grabungsreihe relativ schnell mit einer geschlossenen Schuttlage überdeckt werden, so daß nur noch vereinzelt das Röt frei lag. Die Oberhangneigung dürfte auch eine ent- 
scheidende Rolle für den Abfluß der Niederschläge gespielt haben. An steileren Gehängen fließt das Schmelzwasser schneller ab und entfaltet eine größere Transportkraft, während es an Flachhängen im Detritus versickert und diesen durchtränkt. Hierin mag die Ursache dafür liegen, daß in dem einen Falle die Abspülung, in dem anderen die Solifluktion dominierte. Beobachtungen von MORTENSEN (1930) und BüDEL (1960) aus den rezenten Periglazialgebieten zeigen, daß dort das Verhältnis von Abspülung und Solifluktion bei gleichen klimatischen Gegebenheiten in starkem Maße von der Geländeneigung abhängt. Die verschiedene Genese der beiden Schichten ließe sich also auf die unterschiedliche Oberhangneigung zurückführen.

Tabelle 4

Stratigraphische Übersicht über Schuttdeckenfolgen an Muschelkalkkämmen und -stufen in Südniedersachsen

\begin{tabular}{|c|c|c|c|c|}
\hline \multicolumn{2}{|c|}{ Weper nordwestlich Göttingen ${ }^{1}$ ) } & \multicolumn{2}{|c|}{ Göttinger Wald } & \multirow[b]{2}{*}{$\begin{array}{c}\text { SZ-Lichtenberg } \\
\text { Baugrube }\end{array}$} \\
\hline $\begin{array}{l}\text { Grabungsreihe } \\
\text { Leisenrode }\end{array}$ & $\begin{array}{l}\text { Bauaufschluß } \\
\text { in Hardegsen }\end{array}$ & $\begin{array}{l}\text { Grabenprofil } \\
\text { Eddigehausen }\end{array}$ & $\begin{array}{l}\left.\text { Gr. Lengden }{ }^{2}\right) \\
\text { Bauaufschluß }\end{array}$ & \\
\hline Solifluktionslöß & Solifluktionslöß & Solifluktionslöß & & Solifluktionslöß \\
\hline $\begin{array}{l}\text { lößfreier Muschel- } \\
\text { kalkwanderschutt }\end{array}$ & $\begin{array}{l}\text { lößhaltiger } \\
\text { Muschelkalk- } \\
\text { wanderschutt }\end{array}$ & $\begin{array}{l}\text { lößfreier Muschel- } \\
\text { kalkwanderschutt }\end{array}$ & $\begin{array}{l}\text { lößfreier Muschel- } \\
\text { kalkwanderschutt }\end{array}$ & \multirow{2}{*}{$\begin{array}{l}\text { lößfreier } \\
\text { Muschelkalk- } \\
\text { wanderschutt }\end{array}$} \\
\hline Rötfließerde & Rötfließerde & Rötfließerde & Rötfließerde & \\
\hline \multirow{2}{*}{$\begin{array}{l}\text { lößhaltiger } \\
\text { Muschelkalk- } \\
\text { wanderschutt }\end{array}$} & $\begin{array}{l}\text { muschelkalk- } \\
\text { haltiger Löß }\end{array}$ & \multirow{2}{*}{$\begin{array}{l}\text { lößhaltiger } \\
\text { Muschelkalk- } \\
\text { wanderschutt }\end{array}$} & \multirow[t]{2}{*}{$\begin{array}{l}\text { muschelkalk- } \\
\text { haltiger Löß }\end{array}$} & \multirow{2}{*}{$\begin{array}{l}\text { lößhaltiger } \\
\text { Muschelkalk- } \\
\text { schwemmschutt }\end{array}$} \\
\hline & \multirow{2}{*}{$\begin{array}{l}\text { Röt-Muschelkalk- } \\
\text { Fließerde }\end{array}$} & & & \\
\hline \multirow{2}{*}{$\begin{array}{l}\text { Röt-Muschelkalk- } \\
\text { Fließerde }\end{array}$} & & \multirow[t]{2}{*}{ Rötfließerde } & \multirow[t]{2}{*}{ Rötfließerde } & \multirow[t]{2}{*}{ Keuperfließerde } \\
\hline & \multirow{2}{*}{$\begin{array}{l}\text { Schwemmsedi- } \\
\text { mente aus Löß, } \\
\text { Muschelkalk- und } \\
\text { Rötdetritus }\end{array}$} & & & \\
\hline & & $\begin{array}{l}\text { 1) aus } \mathrm{RoH} \\
\text { 2) freundl. }\end{array}$ & $\begin{array}{l}\text { ENBURG 1965a. } \\
\text { ündl. Mitteilun }\end{array}$ & Dr. ROHDENBURG. \\
\hline
\end{tabular}

Im Hildesheimer Wald liegt also unter einer Lößdecke ein lößfreier Muschelkalkwanderschutt oder eine Folge Muschelkalkwanderschutt-Rötfließerde, die von einer teilweise lößhaltigen Serie unterlagert werden. Diese älteren Lockersedimente bestehen unterhalb steilerer Hänge vielfach aus Schwemmschutt, in flacheren Partien größtenteils aus solifluidalen Bildungen. Ahnlich aufgebaute Schuttdeckenfolgen wurden auch an anderen Punkten im niedersächsischen Bergland beobachtet (Tab. 4). Diesen Hangsedimenten ist gemeinsam, daß eine Lößdecke einen meist lößfreien Muschelkalkwanderschutt oder eine Folge Muschelkalkwanderschutt-Rötfließerde überdeckt. Da sich diese Serie immer wieder in derselben Ausbildung feststellen ließ, dürften diese zwei bzw. drei Schichten einander etwa entsprechen und mit dem Löß, dem Muschelkalkwanderschutt und der Rötfließerde im Hildesheimer Wald zu parallelisieren sein. Der basale Teil der in Tab. 4 wiedergegebenen Profile zeigt stets in einzelnen Lagen eine Lößbeimengung. Außerdem treten darin vielfach Schwemmsedimente auf. Diese Schichten scheinen also in Teilen oder als Ganzes den älteren Sedimenten im Hildesheimer Wald zu entsprechen. Da sich Schwemmsedimente in bodenstratigraphisch gliederbaren Profilen vorwiegend in einen frühen Abschnitt der letzten Kaltzeit stellen lassen, können die Schwemmsedimente im Hildesheimer Wald, an der Weper und in den Lichtenbergen aus dem Altwürm oder dem Mittelwürm (im Sinne von Woldstedt 1962) stammen. Einen exakten Beweis für diese Datierung gibt es jedoch nicht. 
Merkwürdigerweise treten saaleglaziale glazigene Ablagerungen nur selten an den untersuchten Hängen auf. Sie müssen also nach dem drenthezeitlichen Eisvorstoß, aber vor Ablagerung der beobachteten würmzeitlichen Sedimente abgetragen worden sein. Diese Abtragungsphase kann nicht mit dem Eeminterglazial gleichgesetzt werden, denn während der Eemwarmzeit dürfte die flächenhafte Abtragung der Strukturformen des Hildesheimer Waldes analog den holozänen Verhältnissen stagniert haben. Es spricht daher mehr dafür, daß die saalezeitlichen Hangsedimente in einer Spätphase des Saaleglazials oder zu Beginn der Weichselkaltzeit entfernt wurden.

Diese Uberlegungen zur Stratigraphie stellen nur eine Arbeitshypothese dar. Mit mehr Sicherheit lassen sich jedoch geomorphologische Schlußfolgerungen aus der Beschaffenheit der Schuttdecken ziehen.

In dem Überwiegen der Solifluktionshorizonte kommt das Vorherrschen der solifluidalen Abtragung zum Ausdruck. Die vor allem in den selten aufgeschlossenen unteren Hangpartien zu beobachtenden Schwemmsedimente zeigen jedoch, daß auch der Abspülung größere Bedeutung beizumessen ist. Die Schwemmsedimente finden sich in dem Profil 1 in flächenhafter Verbreitung, sind bei Salzgitter-Lichtenberg in eine breite Mulde eingelagert und füllen bei Hardegsen relativ schmale Mulden. Es lassen sich also Übergänge von flächenhafter zu linienhafter Abtragung feststellen. Aus der teilweise $4 \mathrm{~m}$ betragenden Mächtigkeit läßt sich die Intensität der Abspülung ablesen. Sie dürfte sogar noch größer gewesen sein, als man aufgrund der Schuttmächtigkeit annehmen sollte, da der Schwemmschutt bis in die unmittelbare Nähe des subsequenten Baches reicht. Entsprechend der größeren Transportweite bei aquatischer Verfrachtung ist sicher ein großer Teil der Schwemmsedimente in das subsequente Tal gelangt und heute nicht mehr faßbar.

Nur durch diesen aquatischen Transport ist der hohe Muschelkalkgehalt der kaltzeitlichen Schotterkörper innerhalb des Hildesheimer Waldes zu erklären, denn der Muschelkalkwanderschutt gewinnt nur selten Anschluß an die subsequenten Seitenäste der Hauptentwässerungslinien und dürfte die kaltzeitlichen Gewässer nur in geringem Maße mit Kalkschutt beliefert haben.

Die Wellenkalkstirnhänge des Hildesheimer Waldes und der Stufenhang der Weper (RohDENBuRg 1965a) sind im großen und ganzen Vorzeitformen, wie der Würmlöß zeigt, der die Hänge teilweise überzieht. Das an Strukturformen des Wellenkalkes häufig auftretende konkave Stirnhangprofil kann daher nicht, wie es MorTENSEN (1960) für den Göttinger Wald annimmt, stets eine Folge holozäner grundwasserbedingter Abtragung sein. Morphologisch wirksam waren im Hildesheimer Wald und an der Weper nur die kaltzeitlichen Vorgänge. Da die Rötfließerden meistens eine größere Mächtigkeit besitzen als der Muschelkalkwanderschutt, muß der Unterhang stärker abgetragen worden sein als der Oberhang. Der Schwerpunkt der Abtragung muß in der Nähe der Röt-Muschelkalk-Grenze gelegen haben, denn die älteren Schuttdecken fehlen hier. Infolge der stärkeren Unterhangabtragung und der Rückverlegung der Röt-Muschelkalk-Grenze wurde der Unterhang verflacht und der Oberhang versteilt, so daß sich die Durchbiegung des Hanges vergrößerte. Die pleistozän-kaltzeitlichen Prozesse zeigen also eine Tendenz zur Herausbildung konkaver Wellenkalkstirnhänge.

\section{Literaturverzeichnis}

Ackermann, E.: Zur Unterscheidung glazialer und postglazialer Fließerden. Geol. Rdsch. 43, 328-341, Stuttgart 1955. - - Der Abtragungsmechanismus bei Massenverlagerungen an der Wellenkalkschichtstufe. Z. Geomorph. 3, 193-226, 283-304, Berlin 1959.

Bartels, G.: Geomorphologie des Hildesheimer Waldes. Diss. Göttingen 1966.

BLENK, M.: Morphologie des nordwestlichen Harzes und seines Vorlandes. Göttinger geogr. Abh. 24, 1960. 
BüDEL, J.: Die morphologische Wirkung des Eiszeitklimas im gletscherfreien Gebiet. Geol. Rdsch. 34, 482-519, 1944. - - Die Klimaphasen der Würmeiszeit. Naturwiss. 37, 438-449, 1950. - - Die Frostschuttzone Südost-Spitzbergens. Colloquium Geographicum 6, Bonn 1960.

Lürtig, G.: Alt- und mittelpleistozäne Eisrandlagen zwischen Harz und Weser. Geol. Jb. 70, 43-125, Hannover 1954.

Mortensen, H.: Einige Oberflächenformen in Chile und auf Spitzbergen im Rahmen einer vergleichenden Morphologie der Klimazonen. Peterm. geogr. Mitt., Erg. H. 209, 47-156, Gotha 1930. - - Neues über den Bergrutsch südlich der Mackenröder Spitze und über die holozäne Hangformung an Schichtstufen im mitteleuropäischen Klimabereich. Z. Geomorph. Supplementbd. 1, 114-123, Berlin 1960.

Poser, H.: Dauerfrostboden und Temperaturverhältnisse während der Würmeiszeit im nicht vereisten Mittel- und Westeuropa. Naturwiss. 34, 10-18, 1947. - - Die nördliche Lößgrenze in Mitteleuropa und das spätglaziale Klima. Eiszeitalter u. Gegenw. 1, 27-55, Öhringen 1951.

Poser, H. \& HövermanN, J.: Untersuchungen zur pleistozänen Harzvergletscherung. Abh. braunschw. wiss. Ges. 3, 61-115, 1951.

Rohdenburg, H.: Die Muschelkalk-Schichtstufe am Ostrand des Sollings und Bramwaldes. Göttinger geogr. Abh. 33, 1965a. - - Untersuchungen zur pleistozänen Formung am Beispiel der Westabdachung des Göttinger Waldes. Gießener geogr. Schr. 7, 1965b.

Rohdenburg, H. \& Meyer, B.: Rezente Mikroformung in Kalkgebieten durch inneren Abtrag und die Rolle der periglazialen Gesteinsverwitterung. Z. Geomorph. 7, 120-146, Berlin 1963.

Schönhals, E., Rohdenburg, H. \& Semmel, A.: Ergebnisse neuerer Untersuchungen zur Würmlöß-Gliederung in Hessen. Eiszeitalter u. Gegenw. 15, 199-206, Öhringen 1964.

SfönEmanN, J.: Geomorphologische Untersuchungen an Schichtkämmen des Niedersächsischen Berglandes. Göttinger geogr. Abh. 36, Göttingen 1966.

Woldstedt, P.: Uber die Gliederung des Quartärs und Pleistozäns. - Eiszeitalter u. Gegenw. 13, 115-124, Öhringen 1962.

Manuskr. eingeg. 20. 9. 1966.

Anschrift des Verf.: Gerhard Bartels, 34 Göttingen, Gerichtslinde 1. 